University of Nebraska - Lincoln

DigitalCommons@University of Nebraska - Lincoln

$1-1-2000$

\title{
Excitation and damping of a self-modulated laser wakefield
}

Shouyuan Chen

University of Michigan, schen6@unl.edu

M. Krishnan

University of Michigan

Anatoly Maksimchuk

University of Michigan, tolya@umich.edu

Donald P. Umstadter

University of Nebraska-Lincoln, donald.umstadter@unl.edu

Follow this and additional works at: https://digitalcommons.unl.edu/physicsumstadter

Part of the Physics Commons

Chen, Shouyuan; Krishnan, M.; Maksimchuk, Anatoly; and Umstadter, Donald P., "Excitation and damping of a self-modulated laser wakefield" (2000). Donald Umstadter Publications. 4.

https://digitalcommons.unl.edu/physicsumstadter/4

This Article is brought to you for free and open access by the Research Papers in Physics and Astronomy at DigitalCommons@University of Nebraska - Lincoln. It has been accepted for inclusion in Donald Umstadter Publications by an authorized administrator of DigitalCommons@University of Nebraska - Lincoln. 


\title{
Excitation and damping of a self-modulated laser wakefield
}

\author{
S.-Y. Chen, ${ }^{\text {a) }}$ M. Krishnan, A. Maksimchuk, and D. Umstadter \\ Center for Ultrafast Optical Science, University of Michigan, Ann Arbor, Michigan 48109
}

(Received 23 August 1999; accepted 11 October 1999)

\begin{abstract}
Spatially, temporally, and angularly resolved collinear collective Thomson scattering was used to diagnose the excitation and damping of a relativistic-phase-velocity self-modulated laser wakefield. The excitation of the electron plasma wave was observed to be driven by Raman-type instabilities. The damping is believed to originate from both electron beam loading and modulational instability. The collective Thomson scattering of a probe pulse from the ion acoustic waves, resulting from modulational instability, allows us to measure the temporal evolution of the plasma temperature. The latter was found to be consistent with the damping of the electron plasma wave. (C) 2000 American Institute of Physics. [S1070-664X(00)04201-4]
\end{abstract}

\section{INTRODUCTION}

The study of growth and damping of electron plasma waves (EPW) excited during propagation of a high-intensity laser pulse in a plasma is relevant to many applications, such as the fast ignitor concept in laser fusion, ${ }^{1} \mathrm{x}$-ray lasers, and laser-plasma-based electron accelerators. ${ }^{2}$ Two collisionless damping processes have been observed to damp EPWs on a time scale short compared to collisional damping processes. One is electron beam loading, in which hot electrons in the plasma are trapped and heated by the EPW, when their thermal velocities are higher than the trapping threshold. Another is due to the decay of an EPW into an ion acoustic wave (IAW) and daughter EPWs as a result of modulational instability of EPWs. ${ }^{3-5}$ Both have been observed in the damping of the EPW excited by Raman backscattering instability (which is a slow-phase-velocity wave), ${ }^{6-8}$ and the latter have been observed in the collinear laser-beat-wave (which is a fast-phase-velocity wave). ${ }^{9,10}$

The damping of a self-modulated laser wakefield was attributed to electron beam loading by Tzeng et al. ${ }^{11}$ in studies using a particle-in-cell simulation that assumes immobile ions. In this case, the EPW amplitude is not high enough to induce longitudinal (one-dimensional) wavebreaking. They believe the occurrence of Raman backscattering and sidescattering initially heats the electrons such that the latter have sufficient energy to be trapped by the large-phasevelocity $\left(\mathrm{V}_{p} \sim c\right)$ EPW that is generated from Raman forward-like scattering. ${ }^{12-15}$ On the other hand, Bulanov et al. ${ }^{16}$ suggested that transverse wave-breaking can cause damping of a three-dimensional EPW and inject electrons into the trapping region of the plasma wave.

Two groups ${ }^{17,18}$ have investigated the evolution of a self-modulated laser wakefield experimentally by use of collinear collective Thomson scattering and, yet, gave different explanations for the observed fast damping of the EPW. $\mathrm{We}^{17}$ previously attributed the damping of the EPW to acceleration of electrons in the forward direction based on the observation that the total energy carried by these electrons is

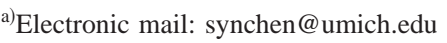

comparable with the energy stored in the EPW at its peak. However, this argument fails if the damping of the EPW through various mechanisms leads to saturation of the EPW during the laser pulse. Ting et al. ${ }^{18}$ observed the growth and decay of the scattered light near the probe wavelength in the $30^{\circ}$ direction (with respect to the direction of beam propagation). They attributed such enhanced scattering of the probe light to the collective scattering from IAWs that are produced by modulational instability decay of the EPW. However, without resolving the scattered light spectrally and angularly, the attribution of such scattering to IAWs is unconvincing and competing mechanisms could not be ruled out.

In this experiment, a self-modulated laser wakefield excited during the propagation of an intense laser pulse in a plasma was observed through the appearance of Raman satellites. Strong spectral cascading (up-to-4th anti-Stokes satellites) was detected, and their relative strengths indicate that a large-amplitude (up to $30 \%$ density perturbation) electron plasma wave was excited. Observation of anti-Stokes satellites indicates strong coupling to a plasma wave driven by a Raman-type instability, either Raman forward scattering or self-modulation instability. ${ }^{19-23}$ In addition, relativistic electrons generated by the electron plasma wave were observed. These results have been reported previously in Ref. 12. In Sec. II, new results for angularly resolved Raman spectra are presented, which allow us to look into the three-dimensional features of Raman scattering instability.

To measure the growth and the decay of the plasma wave, i.e., its temporal distribution, collinear collective Thomson scattering of a second-harmonic probe pulse was used. The growth of the plasma wave is found to be exponential, and the high damping rate limits the duration of the plasma wave to a few picoseconds. These observations have been published previously in Ref. 17, and are summarized in Sec. III. New results for various pump laser powers are discussed in Sec. IV, providing new clues about the dominant damping mechanisms. In Sec. V, we discuss our studies of the spatially, temporally, and angularly resolved collective Thomson spectra to investigate the three-dimensional features and the spatio-temporal distribution of the electron 
plasma wave excited. In Sec. VI, we report the results of our investigation of the damping processes of the self-modulated laser wakefield. The spatio-temporal distribution of the IAW was measured using collinear collective Thomson scattering, revealing the importance of modulational instability in the damping of a self-modulated laser wakefield, in addition to the effect of electron beam loading. The time-varying angularly dependent frequency shifts of collective Thomson scattering satellites-presumably from IAWs-were obtained using angularly resolved spectroscopy, rendering the measurement of the temporal evolution of the effective electron temperature. These results allow us to systematically identify the relative importance of the various damping mechanisms for the self-modulated laser wakefield, and diagnose the subsequent plasma heating. A summary is given in Sec. VII.

\section{OBSERVATION OF RAMAN SCATTERING INSTABILITY VIA ANGLE-RESOLVED RAMAN SCATTERING SPECTRA}

In this experiment, we used a laser system that produced $400 \mathrm{fs}$ laser pulses at $1.054 \mu \mathrm{m}$ wavelength with a maximum peak power of $4 \mathrm{TW}$. The $50 \mathrm{~mm}$ diam laser beam was focused with an $f / 3.3$ harmonically coated parabolic mirror to a small spot of $8 \mu \mathrm{m}$ FWHM (full width at half maximum) in diameter at the front edge of a supersonic helium gas jet. (This small spot contains $60 \%$ of the pulse energy, and the rest $(40 \%)$ is in a large spot of $>100 \mu \mathrm{m}$ in diameter.) A co-propagating second-harmonic probe pulse was focused by the same parabolic mirror to a spot of $7 \mu \mathrm{m}$ FWHM, overlapping with the pump-beam focus. ${ }^{17}$ When a flat-topped gas jet $(1000 \mu \mathrm{m}$ in length) with a sharp gradient $(250 \mu \mathrm{m})$ was used, relativistic-ponderomotive self-channeling of the pump pulse was observed when the peak laser power was $\geqslant 1.5 \mathrm{TW}$ for $3.7 \times 10^{19} \mathrm{~cm}^{-3}$ plasma density. ${ }^{24,25}$ Under such conditions, the probe pulse was observed to be guided by the plasma-density depression created by the selfchanneled pump pulse. ${ }^{25,26}$

To measure the spectra of Raman scattering in several directions simultaneously, glass fibers of $1 \mathrm{~mm}$ core diameter were set up $2 \mathrm{~cm}$ away from the laser focus, i.e., $f / 20$ acceptance, with each fiber looking from a specific angle. The opposite ends of the fibers were lined up on the slit of a prism spectrometer. The prism spectrometer has a resolution of $\lambda / \Delta \lambda=300$ at $\lambda=527 \mathrm{~nm}$ and $\lambda / \Delta \lambda=600$ at $\lambda$ $=1054 \mathrm{~nm}$. A prism spectrometer, instead of a grating spectrometer, was used because of its ability to measure the entire spectral region of interest (including the fundamental and the satellites) in a single shot. However, if the glass fiber is situated within the forward laser cone $\left(-10^{\circ}<\theta<+10^{\circ}\right)$, the strong laser light will damage the fiber tip and also generate white light in the fiber, burying the signal. Furthermore, a higher efficiency of signal collection is needed in the direct forward channel in order to increase the sensitivity of measurement of plasma wave amplitude. Therefore, whenever the measurement of the spectrum in the direct forward direction $\left(0^{\circ}\right)$ was needed, an imaging system with lenses $(f / 15)$ was used instead. The setup is shown in Fig. 1.

The direct forward scattering spectra $\left(\right.$ at $\left.0^{\circ}\right)$ were re-

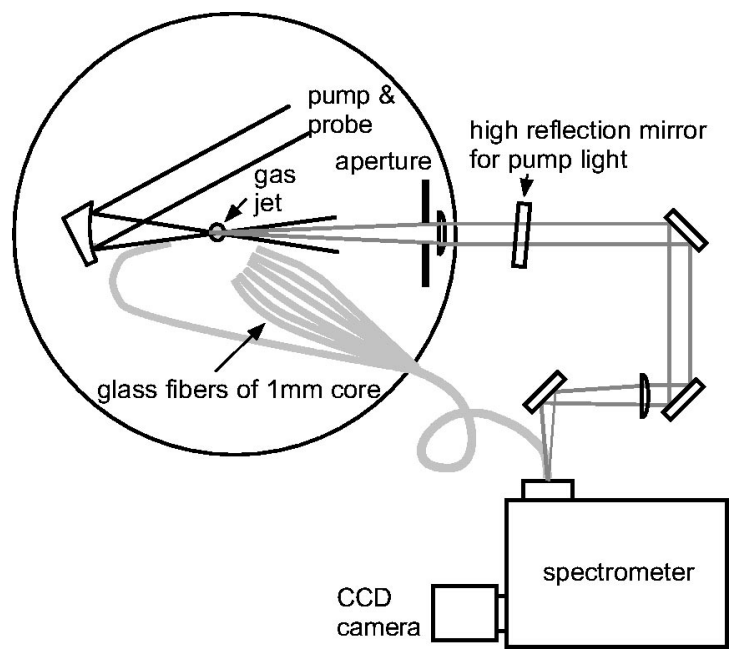

FIG. 1. Setup of angle-resolved spectroscopy for detecting the spectra of Raman scattering or collective Thomson scattering. The fiber array was removed from the entrance slit of the spectrometer when the direct forward channel (using lenses) was used. The spectrometer was a prism spectrometer when a low spectral resolution and a large range were needed, and was a 1200 lines/mm grating spectrometer when a high spectral resolution was needed.

ported in Ref. 12 for a shorter and nonuniform gas jet (400 $\mu \mathrm{m}$ long), with which relativistic-ponderomotive selfchanneling was not observed. Here we discuss the results obtained by using the spectra measured in the $15^{\circ}$ channel for the $1 \mathrm{~mm}$ long uniform gas jet. All qualitative features were found to be the same as in the results of the $0^{\circ}$ channel for that $400 \mu \mathrm{m}$ long gas jet (see Ref. 12). Figure 2 shows the Raman spectra in the $15^{\circ}$ channel for various plasma densities at $2.5 \mathrm{TW}$ pump power, and Fig. 3 shows the results for various laser powers at $3.7 \times 10^{19} \mathrm{~cm}^{-3}$ plasma density. The dependence of the anti-Stokes frequency shift on plasma density is consistent with the theoretical prediction

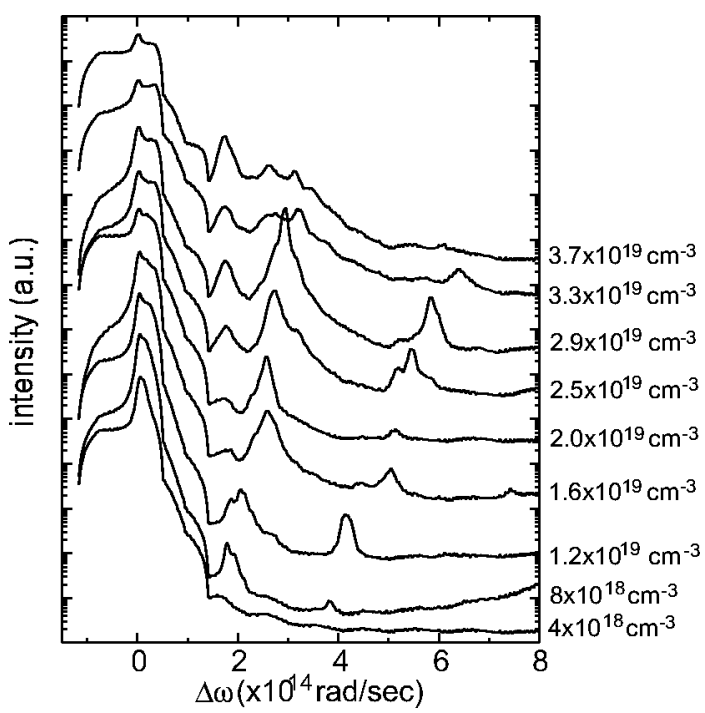

FIG. 2. Spectra of Raman forward scattering in the $15^{\circ}$ direction for various plasma densities at a fixed laser power of $2.5 \mathrm{TW}$. Note that the intensity scale is logrithmic and that the curves are vertically displaced for ease of viewing. 


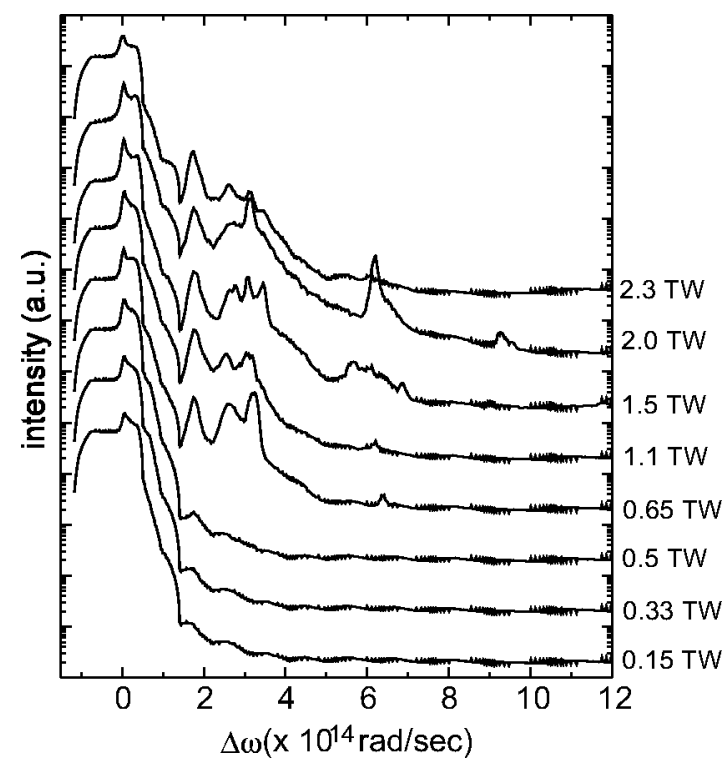

FIG. 3. Spectra of Raman forward scattering in the $15^{\circ}$ direction for various laser powers at a fixed plasma density of $3.7 \times 10^{19} \mathrm{~cm}^{-3}$. Note that the intensity scale is logrithmic and that the curves are vertically displaced for ease of viewing.

$$
\omega_{p}(\gamma)=\omega_{p 0} / \sqrt{\gamma}
$$

where

$$
\omega_{p 0}=\sqrt{4 \pi n_{e} e^{2} / m_{e}}=5.64 \times 10^{4} n_{e}^{1 / 2}\left[\mathrm{~cm}^{-3}\right] \mathrm{rad} / \mathrm{s},
$$

is the nonrelativistic plasma frequency which is determined by electron density $n_{e}$ only and

$$
\gamma=\sqrt{1+\frac{a_{0}^{2}}{2}}
$$

is the relativistic factor associated with the average transverse quivering velocity of an electron in a laser field; $a_{0}$ is the normalized vector potential of the laser field. The fact that such relation is obeyed, i.e., $\omega_{p} \propto \sqrt{n_{e}}$, proves that the satellites observed are indeed Raman scattering satellites. Strong spectral cascading was observed (up to the fourth anti-Stokes satellite), indicating strong coupling to a plasma wave generated by Raman forward scattering and selfmodulation instabilities. ${ }^{19-23}$ The amplitude of the plasma wave, which is proportional to the square root of the relative strength between the anti-Stokes signal and the fundamental, increases with increase of laser power and plasma density, as expected from the temporal growth rate for Raman forward scattering ${ }^{11}$

$$
\gamma_{0}=\frac{\omega_{p}^{2}}{\sqrt{8} \omega_{0}} \frac{a_{0}}{\sqrt{1+a_{0}^{2} / 2}},
$$

where $\omega_{0}$ is the laser frequency.

Note that the Raman satellites become broadened at high laser power and plasma density. Such broadening may be a result of relativistic self-phase modulation of the pump pulse and its relativistic cross-phase modulation on the Raman satellites. Another possibility is the loss of the coherence of the plasma wave as a result of strong damping via, e.g., strong

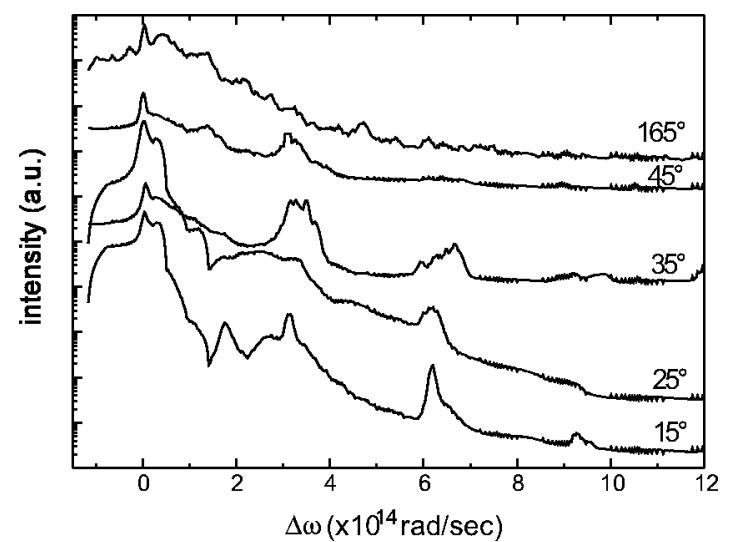

FIG. 4. Spectra of Raman forward scattering for various angles of observation at $2.5 \mathrm{TW}$ laser power and $3.7 \times 10^{19} \mathrm{~cm}^{-3}$ plasma density. Note that the intensity scale is logrithmic and that the curves have been vertically displaced for ease of viewing.

beam loading (or wave-breaking), leading to a turbulent plasma. The scattering would then be due to the individual electrons and not due to a single collective mode of plasma. Since the electrons are still bunched in space ( $k$-matched) but now have a range of velocities, they will produce a broadened spectrum. ${ }^{12,27,28}$ This process is referred to as stimulated Compton scattering, ${ }^{29,30}$ which has a large $k \lambda_{\text {De }}$ in contrast to stimulated Raman scattering.

The Raman spectra at various angles are shown in Fig. 4 for a laser power of $2.5 \mathrm{TW}$ and a plasma density of 3.7 $\times 10^{19} \mathrm{~cm}^{-3}$. The spectra in all channels of forward directions show roughly the same features. As can be seen, the intensities of the Raman satellites decrease more slowly with increasing angle than the fundamental does. This shows that the angular spread of the Raman satellites is larger than that of the fundamental, which is indicative of the small cross section of the plasma wave, in addition to the small cross section of the pump beam. On the other hand, near-forward Raman scattering and Raman side-scattering instabilities may also contribute to the observed satellites at larger angles. The Stokes satellites were not measured because the CCD (charge-coupled device) camera is not sensitive in that spectral range.

The spectrum measured in the $165^{\circ}$ channel shows a strongly modulated spectrum extending from the red side of the fundamental wavelength to the blue side (higher than the second anti-Stokes satellites). Normally, for stimulated Raman backscattering, only Stokes is resonant. The appearance of the signal on the blue side is believed to be a result of both strong broadening and blue shift of the first Stokes satellite as a result of Raman backscattering in the strong coupling regime, as discussed in Ref. 31 .

\section{MEASUREMENT OF PLASMA WAVE AMPLITUDE USING COLLINEAR COLLECTIVE THOMSON SCATTERING}

Measurements of Raman scattering spectra can provide much information, such as the plasma density, the existence of an electron plasma wave, and a rough estimate of peak plasma-wave amplitude. However, in order to properly un- 


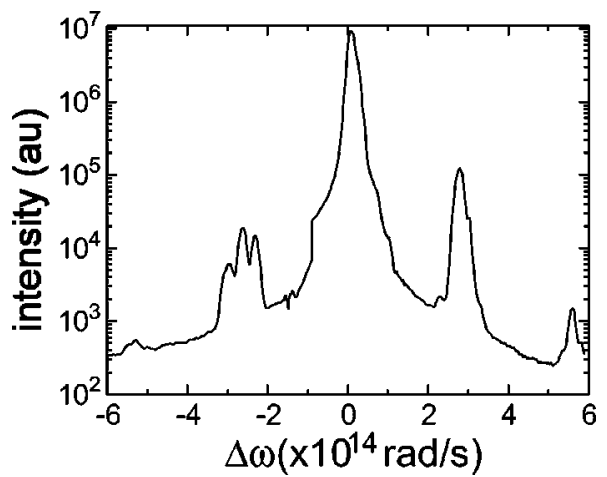

FIG. 5. Direct forward Thomson scattering spectrum for $3 \mathrm{TW}$ pump power, $2.3 \times 10^{19} \mathrm{~cm}^{-3}$ plasma density, and zero probe delay.

derstand the growth and decay of the electron plasma waves generated, it is necessary to characterize the distribution of the plasma-wave amplitude spatially and temporally. To measure the growth and decay of the electron plasma wave excited by Raman forward scattering and self-modulation instabilities, collinear collective Thomson scattering was used. When the second-harmonic probe pulse propagated collinearly with the pump pulse in the plasma, it was scattered by the plasma wave to longer wavelengths (Stokes Thomson satellites) and shorter wavelengths (anti-Stokes Thomson satellites), i.e., $\omega_{s}=\left(2 \omega_{0}\right) \pm n \cdot \omega_{p}$, where $n$ is the order of the satellite.

In the first step, the spatially-averaged temporal distribution of the EPW was measured. Forward scattered light from the probe pulse was collected on-axis $\left(0^{\circ}\right)$ and spectrally resolved by using the prism spectrometer. Since the focus of the probe beam overlapped with the focus of the pump beam and the former is slightly smaller than the latter, the probe pulse measured a plasma wave amplitude that is averaged spatially over the probe spot size transversely and the channel length of the plasma wave longitudinally.

The results for a $400 \mu \mathrm{m}$ long nonuniform gas jet were reported in Ref. 17 and are summarized as follows. Figure 5 shows the Thomson scattering spectrum for zero probe delay (i.e., when the pump pulse and the probe pulse overlap temporally) for a pump-pulse peak power of $3 \mathrm{TW}$ and a plasma density of $2.3 \times 10^{19} \mathrm{~cm}^{-3}$. Two Stokes and anti-Stokes satellites were detected, and, from their amplitudes, the plasmawave amplitude can be determined. The ratio between the intensities of Thomson satellites and the incident probe can be used to determine the plasma wave amplitude by applying the Bragg scattering equation ${ }^{32}$

$$
\frac{P_{s}}{P_{0}}=\frac{1}{4} \Delta n^{2} r_{0}^{2} \lambda_{\mathrm{pr}}^{2} L^{2} \frac{\sin ^{2}(\Delta K L)}{(\Delta K L)^{2}},
$$

where $\Delta n$ is the plasma wave amplitude (assuming a sinusoidal plasma wave with frequency $\omega_{p}$ and wave number $\left.k_{p}\right), r_{0}$ is the classical electron radius, $\lambda_{\mathrm{pr}}$ is the probe wavelength, $L$ is the plasma-wave channel length, and $\Delta K=k_{\mathrm{pr}}$ $-k_{s} \pm k_{p}$ is the wave vector mismatch. Assuming a plasmawave channel length of $220 \mu \mathrm{m}$, estimated from the side imaging of the linear Thomson scattering of the pump pulse, the Stokes satellite indicates a plasma-wave amplitude of
$3.6 \%$, while the anti-Stokes satellite indicates $9.2 \%$. However, since $L$ is not actually measured but roughly estimated from the pump-pulse channel length, the obtained plasmawave amplitude has some uncertainty.

There is another approach to calculate the plasma wave amplitude. ${ }^{33-36}$ For a significant plasma-wave amplitude, the plasma-wave density profile steepens and thus contains harmonic components. ${ }^{37,38}$ The harmonics in the plasma density profile can be expanded in terms of the fundamental density perturbation, $\Delta n_{1} / n_{0}$

$$
\frac{\Delta n_{m}}{n_{0}}=\alpha_{m}\left(\frac{\Delta n_{1}}{n_{0}}\right)^{m},
$$

where $m$ is the harmonic number, and the coefficient $\alpha_{m}$ in a cold plasma is

$$
\alpha_{m}=\frac{m^{m}}{2^{m-1} m !} .
$$

Thus, the first two coefficients are $\alpha_{2}=1.0$ and $\alpha_{3}=1.1$. The $m$ th harmonic component of the plasma wave produces the $m$ th Stokes and anti-Stokes satellites. By combining Eqs. (5) and (6), we can obtain the ratio between the intensities of the satellites

$$
\frac{P_{s 2}}{P_{s 1}}=\alpha_{2}^{2}\left(\frac{\Delta n_{1}}{n_{0}}\right)^{2} \frac{\operatorname{sinc}^{2}\left(\Delta K_{2} L\right)}{\operatorname{sinc}^{2}\left(\Delta K_{1} L\right)} .
$$

Since in our results the second Stokes and anti-Stokes satellites are visible, the harmonic analysis can be used and yields amplitudes of $17 \%$ and $12 \%$, respectively. As can be seen, both methods give the same amplitude, $\sim 10 \%$, when the anti-Stokes satellites are used. The results from the Stokes satellites are very different when different methods are used; however, the average result is $\sim 10 \%$, the same as the antiStokes results. Therefore, the anti-Stokes satellites are used for the determination of the plasma wave amplitude in this experiment.

Figure 6 shows the collective Thomson scattering spectra as a function of pump-laser power and plasma density for zero probe delay. ${ }^{17}$ All the features, such as the shift of satellite frequency with plasma density, and the broadening of satellites at high laser power and plasma density, are consistent with those of Raman satellites. The temporal evolution of the plasma-wave amplitude can be obtained by scanning the probe delay. Figure 7 shows the Thomson scattering spectra at different probe delays, and Fig. 8 shows the temporal distribution of the plasma wave amplitude derived from it. The growth and decay of the plasma wave can be fit fairly with exponential functions, except for the region around the peak. The growth rate is measured to be 3.5 $\pm 0.3 \mathrm{ps}^{-1}$ from the Stokes satellites, and $3.3 \pm 0.3 \mathrm{ps}^{-1}$ from the anti-Stokes satellites. The decay rate is measured to be $1.6 \pm 0.1 \mathrm{ps}^{-1}$ and $1.9 \pm 0.2 \mathrm{ps}^{-1}$, respectively.

\section{SPATIALLY AVERAGED TEMPORAL EVOLUTION OF THE PLASMA WAVE AMPLITUDE}

In the recent experiment, we used the $1 \mathrm{~mm}$ long uniform gas jet to repeat the same measurement described in Sec. III under various pump laser powers in order to inves- 

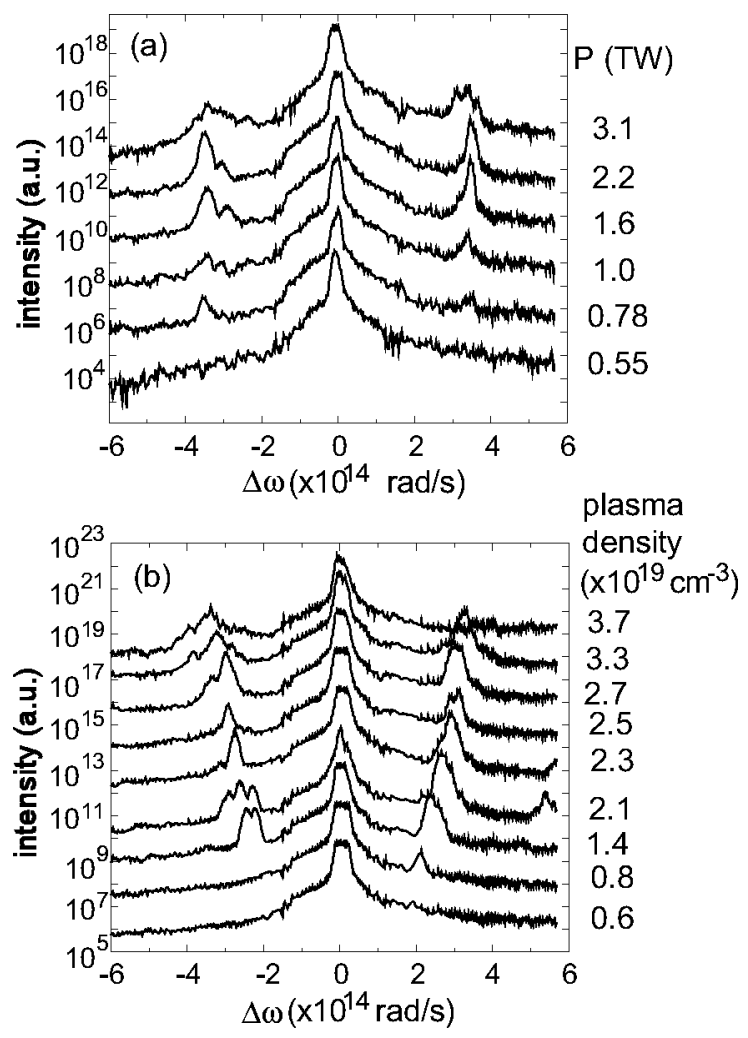

FIG. 6. Direct forward Thomson scattering spectra for various pump powers at $3.7 \times 10^{19} \mathrm{~cm}^{-3}$ plasma density, (a), and for various plasma densities at 3 TW pump power, (b). The probe delay is zero. Note that the intensity scale is logrithmic and that the curves are vertically displaced for ease of viewing.

tigate the damping mechanisms that cause the decay of the EPW. Since the acceleration of electrons by the plasma wave (electron beam loading) can be an important factor for the damping of the plasma wave, the characteristics of the electron beam generated was also monitored in this experiment. ${ }^{12}$ The temporal distribution of the EPW was

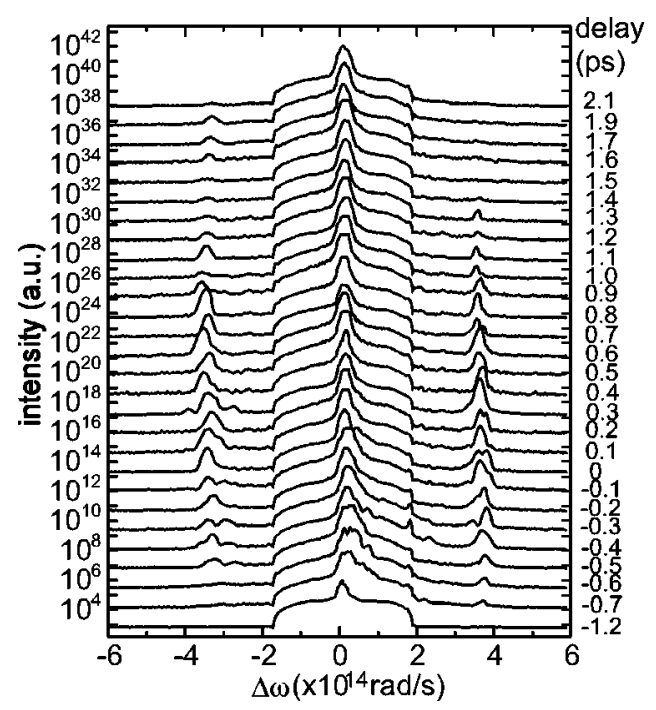

FIG. 7. Direct forward Thomson scattering spectra for various probe delays at $1.5 \mathrm{TW}$ pump power and $3.7 \times 10^{19} \mathrm{~cm}^{-3}$ plasma density. Note that the intensity scale is logrithmic and that the curves are vertically displaced for ease of viewing.

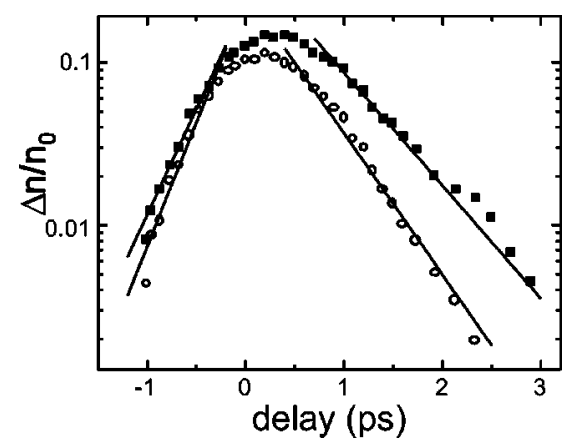

FIG. 8. Amplitude of the plasma wave as a function of probe delay at 1.5 TW pump power and $3.7 \times 10^{19} \mathrm{~cm}^{-3}$ plasma density. Solid squares (open circles) represent the amplitudes derived from anti-Stokes (Stokes) satellites. The exponential fits of the growth and decay rates are also shown.

measured at various laser powers, at which different numbers of electrons were trapped and accelerated. ${ }^{12,24}$ These accelerated electrons propagated in a narrow cone $\left(<20^{\circ}\right.$ FWHM) along the direction of propagation of the laser beam. Figure 9 shows the spatially averaged temporal distribution of the EPW amplitude for various laser powers. The exponential growth rate is measured to be $2.4 \mathrm{ps}^{-1}$ at $1 \mathrm{TW}$, $4.4 \mathrm{ps}^{-1}$ at $1.9 \mathrm{TW}$, and $5.2 \mathrm{ps}^{-1}$ at $3.2 \mathrm{TW}$. At high laser powers, the growth of the EPW begins to saturate, as observed from the shift of the position of the amplitude peak towards earlier times. The EPW damps quickly after the passage of the laser pulse with a damping rate that is roughly $1.8 \mathrm{ps}^{-1}$ at laser power below $1 \mathrm{TW}$ and that rate increases slightly with increasing laser power.

The increase of the growth rate with increase of laser power, shown in Fig. 9, is consistent with the prediction of the theory of Raman forward scattering and self-modulation instabilities. An accurate comparison between the experimental results and the theoretical predictions is difficult because of the complicated evolution of Raman forward scattering and self-modulation instabilities as the pump-pulse propagates in the plasma and the variation of laser intensity with time in a laser pulse. In addition, the actual growth rate is strongly affected by the occurrence of relativistic-

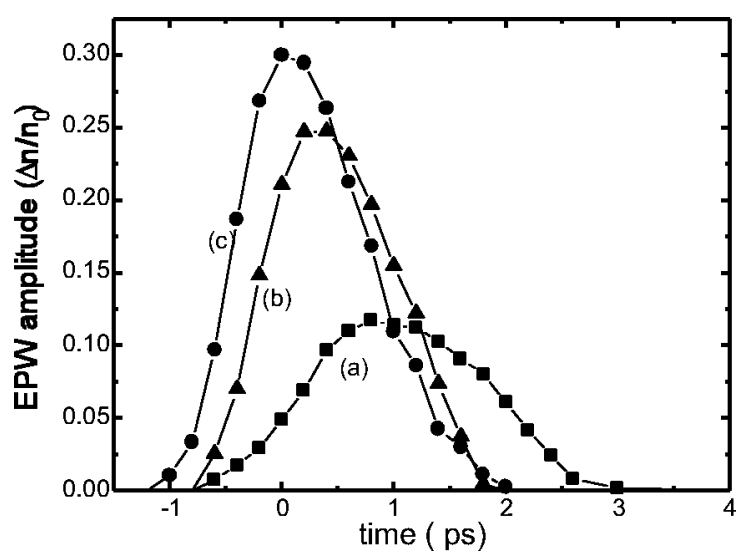

FIG. 9. Spatially averaged temporal evolution of the EPW amplitude for various laser powers at a plasma density of $3.7 \times 10^{19} \mathrm{~cm}^{-3}$ : (a) $1 \mathrm{TW}$, (b) $1.9 \mathrm{TW}$, and (c) $3.2 \mathrm{TW}$. The number of electrons accelerated in each case is $10^{7}, 10^{8}$, and $10^{9}$, respectively. 
ponderomotive self-channeling. ${ }^{13,39-41}$ A simple comparison is the relative temporal growth rates [Eq. (4)] for different pump powers. The relative growth rates are 1:1.4:1.8 at the beginning of the growth (during which $a_{0}^{2} \ll 1$ ) using Eq. (4), and are 1:1.8:2.2 in the experiment. This is reasonably consistent. The actual growth rate and the absolute plasma wave amplitude depend on seeding of the initial plasma wave, which in this case is probably dominated by the ionization effect. Using the equation given by Mori et al. ${ }^{23,42}$

$$
\frac{\Delta n}{n_{0}}=\frac{a_{0}^{2}\left(\psi_{\text {ioniz }}\right)}{4},
$$

where $a_{0}\left(\psi_{\text {ioniz }}\right)$ is the laser-strength parameter at the ionization intensity, the seeding plasma wave amplitude is 4 $\times 10^{-4}$ and $2 \times 10^{-3}$ for singly and doubly ionized helium, respectively. However, the experimental results indicate that the plasma-wave amplitude at the time of ionization $(\sim T$ $-0.7 \mathrm{ps})$ is about $1 \times 10^{-2}$. Therefore, additional seeding is present, such as wake excitation by the front of the laser pulse. The latter can be important if the front of the laser pulse is deformed (sharpened) as a result of its erosion when relativistic-ponderomotive self-channeling occurs ${ }^{43,44}$ or its etching due to Raman sidescattering. . $^{13,23}$

The possible mechanisms that are responsible for the damping of the EPW are discussed in Sec. VI.

\section{SPATIALLY, TEMPORALLY, AND ANGULARLY RESOLVED COLLECTIVE THOMSON SCATTERING}

By using the fiber array setup, the angular distribution of the collective Thomson scattering can be obtained. The intensities of the Thomson satellites were observed to be strongest in the forward direction $\left(0^{\circ}\right)$ and decrease with larger angle, and their angular spreads are larger than the probelaser cone (and the pump-laser cone), similar to the angular distribution of the Raman satellites. One example of the spectra observed at the $15^{\circ}$ channel for various probe delays is shown in Fig. 10. Such large angular spreads of Thomson satellites can be attributed to the small cross section of the pump-laser channel, which results in small cross sections (and thus larger angular spread of wave vectors) of the excited EPW and the guided probe pulse. All forward channels observed similar temporal evolution for Thomson satellites.

If the scattering of the probe beam by the plasma wave in the direction perpendicular to the direction of beam propagation occurs, then direct side imaging of the spatial distribution of the Thomson satellites using matching narrowbandpass filters and a CCD-camera imaging system for various probe delays can measure the spatio-temporal distribution of the amplitude of the plasma wave. Figure 11 shows side images of the first Stokes Thomson satellite at various probe delays. Images of the first anti-Stokes satellite show similar variations. The relative EPW amplitude can be obtained by taking the square root of the ratio between the collective Thomson satellite signal and the probe linear Thomson scattering signal. The temporal duration of the EPW at each position was found to be basically the same as that observed in the spatially averaged measurement (i.e., Fig. 9); therefore, the spatially averaged results can describe

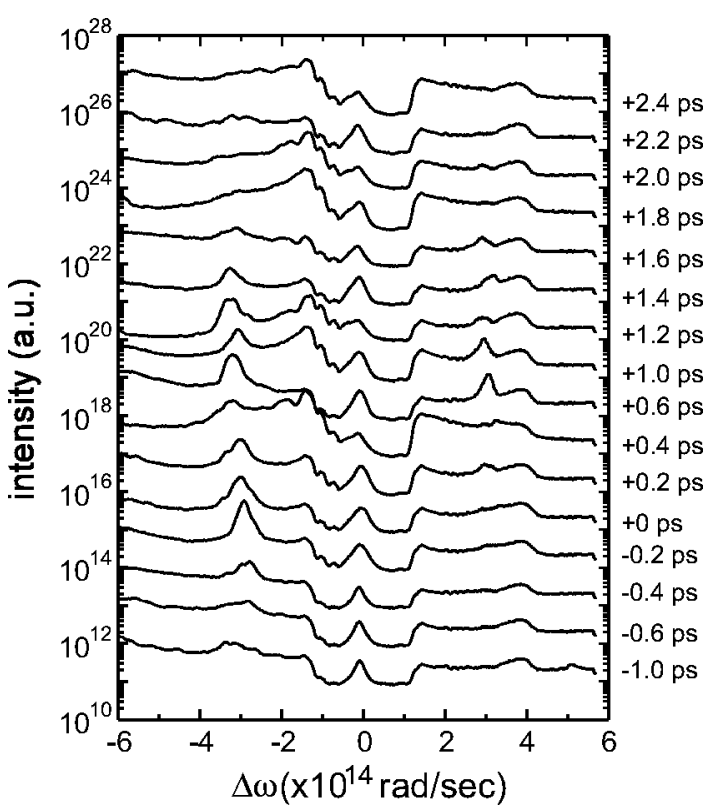

FIG. 10. Spectra of the collective Thomson scattering from the EPW for various probe delays at $15^{\circ}, 2.5 \mathrm{TW}$ pump laser power, and 3.7 $\times 10^{19} \mathrm{~cm}^{-3}$ plasma density. The peak at near $\Delta \omega=4 \times 10^{14} \mathrm{rad} / \mathrm{s}$ is just stray light which does not change with variation of probe delay. The attenuation due to a neutral density filter at the center of the CCD camera chip in this figure is not compensated. Note that the intensity scale is logrithmic and that the curves are vertically displaced for ease of viewing.

the temporal evolution of the plasma wave at every position along the channel. The longitudinal extent of the EPW coincides with that of the pump-pulse and varies accordingly as the pump-pulse undergoes self-channeling. It shows a gradual decrease along the propagation path (probably due to the decrease of pump beam intensity resulting from various loss mechanisms) and contains multiple-peak structure (probably resulting from a multifoci structure in the laser channel).

\section{MECHANISMS FOR DAMPING OF THE ELECTRON PLASMA WAVES}

\section{A. Possible damping mechanisms}

When a relativistic electron beam is generated, acceleration of electrons by the EPW must take energy from the

(a)

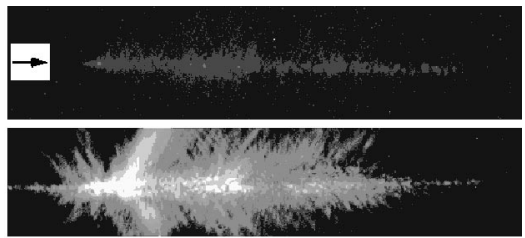

(c)

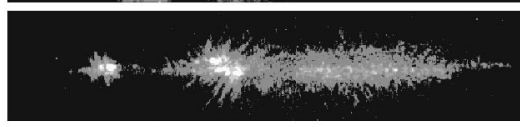

(d)

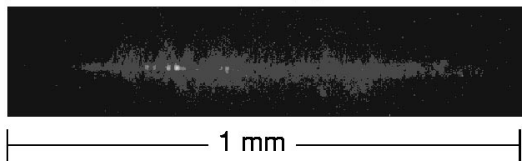

FIG. 11. Side images of the first Stokes-Thomson satellite at various probe delays for $2.5 \mathrm{TW}$ laser power and $3.7 \times 10^{19} \mathrm{~cm}^{-3}$ plasma density: (a) $-1 \mathrm{ps}$, (b) $+0.2 \mathrm{ps}$, (c) $+0.6 \mathrm{ps}$, and (d) $+1.6 \mathrm{ps}$. The arrow indicates the direction of laser propagation. 
EPW and thus should cause damping of the EPW as a result of energy conservation. Therefore, electron beam loading certainly contributes to the saturation and decay of the EPW. At high laser powers, when a large number of electrons are trapped and accelerated, electron beam loading plays a significant role in the saturation of the EPW because the energy carried by these electrons is found to be comparable with the maximum energy stored in the EPW. When this occurs, the energy of the laser pulse that would otherwise be used to drive the EPW is instead diverted to the accelerated electrons and thus the amplitude of the EPW saturates even before the end of the laser pulse. However, the fact that the plasma wave decays at a rate of $1.8 \mathrm{ps}^{-1}$, even when few electrons are accelerated, indicates that at least another damping mechanism takes place besides electron beam loading, and dominates the decay of the EPW at lower laser powers (lower EPW amplitudes).

Modulational instability is a promising candidate for the additional damping mechanism that dominates the decay of the EPW, because it has been observed to dominate the damping of EPWs excited by laser-beat-wave or Raman backscattering. The growth rate of modulational instability is on the order of $\omega_{\mathrm{pi}}$ (ion plasma frequency) in the strong coupling (large EPW amplitude) regime, which, for a 3.7 $\times 10^{19} \mathrm{~cm}^{-3}$ electron density in a fully ionized helium plasma, is $\omega_{\mathrm{pi}} \simeq 5.6 \mathrm{ps}^{-1}$, which is on the same order as the measured EPW decay rate. However, the actual decay rate of the EPW depends on the initial seeding for modulational instability. The initial ion-density fluctuation in this case mainly comes from random thermal fluctuations and hydrodynamic perturbations. This is characteristically different from the decay of the EPWs excited by collinear laser-beatwaves, in which the laser pulses are sufficiently long such that stimulated Brillouin scattering can generate an IAW of significant amplitude with which to seed the modulational instability (and thus lead to discrete wave vectors of the daughter EPWs). ${ }^{9}$ As a result, modulational instability in the case of EPWs generated by short-pulse Raman forward scattering instability (and standard laser wakefield) may or may not be as important as in the long-pulse laser-beat-wave cases and long-pulse Raman backscattering cases. However, modulational instability decay of an EPW can occur when the EPW amplitude exceeds a threshold determined by the IAW damping rate, ${ }^{6}$ and this threshold is generally much lower than that for electron beam loading. Therefore, modulational instability may dominate the decay of the EPW when the EPW amplitude is small.

Two other possible candidates for damping the EPW under our experimental conditions are transverse wave breaking ${ }^{16}$ and the transverse hydrodynamic expansion of the plasma channel. ${ }^{45}$ For transverse wave breaking, the number of plasma oscillation periods before wave breaking occurs should be inversely proportional to the plasma wave amplitude, the self-generated magnetic field strength and the plasma-density depression on axis. ${ }^{16}$ It is, therefore, expected that the duration of the EPW should change significantly with variation of laser power, because these three factors increase with increasing laser power. However, we observed that the EPW decay rates are about the same at 1 and 3 TW

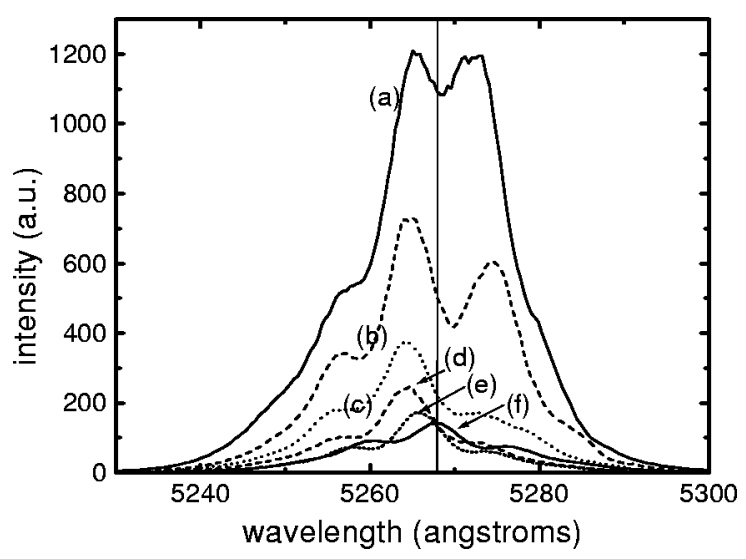

FIG. 12. Spectra of the scattered probe light at various angles for $3 \mathrm{TW}$ laser power, $3.7 \times 10^{19} \mathrm{~cm}^{-3}$ plasma density and 20 ps probe delay: (a) $25^{\circ}$, (b) $35^{\circ}$, (c) $45^{\circ}$, (d) $55^{\circ}$, and (e) $65^{\circ}$. The vertical line indicates the unshifted probe central wavelength. Note that the bumps on the shoulder originate from the original probe spectrum which is monitored with the $165^{\circ}$ channel, (f), which detected the probe light scattered from the nozzle.

laser powers in spite of the occurrence of significant changes in the EPW amplitude (a factor of 3) and the plasma density depression (larger than a factor of 10). Similarly, the damping of the EPW amplitude due to the transverse expansion of the plasma channel should be significantly different for 1 and 3 TW cases. Again, this was not observed in the experiment. Therefore, these two effects, which certainly affect damping of the EPWs to some degree, are not important during the short time scale in which the damping occurs in our experiments.

\section{B. Observations related to modulational instability}

When an EPW is damped by modulational instability, the EPW $\left(\omega_{p}, k_{p}\right)$ is converted into an IAW $(\omega, k)$ and daughter EPWs $\left(\omega_{p} \pm \omega, k_{p} \pm k\right)$. The growth of an IAW upon the decay of the EPW is a signature of this process. To observe the IAWs, collinear collective Thomson scattering of the probe pulse was used in the same way as for the diagnosis of the EPWs, and the results are analyzed in a similar way. In order to resolve the small shift $(\omega)$ of the Thomson satellites from the probe frequency, the fiber array and a 1200 lines $/ \mathrm{mm}$ grating spectrometer (which has a resolution of $0.4 \AA$ at $\lambda=527 \mathrm{~nm}$ ) were used. Four main observations were made and described in the following.

First, the angular dependence of the scattered spectrum was studied. Figure 12 shows the spectra of the scattered probe light at various angles for $3 \mathrm{TW}$ laser power, 3.7 $\times 10^{19} \mathrm{~cm}^{-3}$ plasma density and $20 \mathrm{ps}$ probe delay. Blueshifted and red-shifted peaks were observed and could be the anti-Stokes and Stokes satellites of IAWs, respectively. The small bumps on the shoulder of each peak come from the original spectrum of the probe pulse, as seen in the $165^{\circ}$ channel which monitors the probe backscattered from the nozzle. The asymmetry between the anti-Stokes and Stokes shifts might result from red-shift of the probe due to the decreasing electron density in the channel when selfchanneling occurs, or from the difference between their phase-matching conditions. The Thomson satellites of the 


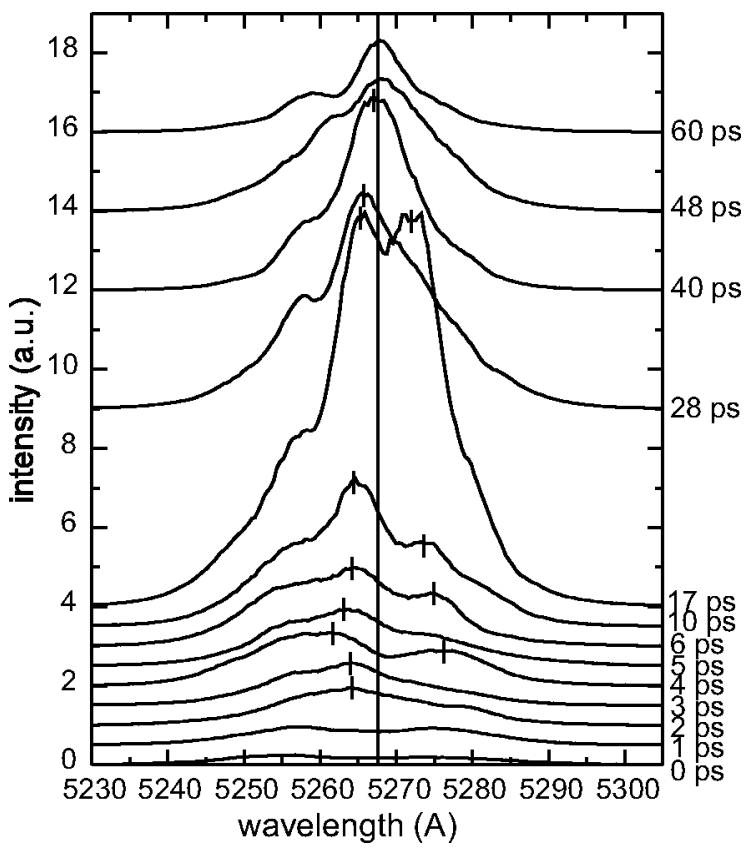

FIG. 13. Spectra of the scattered probe light near the probe wavelength for various probe delays at $25^{\circ}, 3 \mathrm{TW}$ pump laser power, and $3.7 \times 10^{19} \mathrm{~cm}^{-3}$ plasma density. The long vertical line indicates the probe wavelength. The short vertical lines indicate the wavelength of the blue- or red-shifted peaks. Note that the intensity scale is linear and that the curves are vertically displaced for ease of viewing. Also note that the bumps on the shoulder originate from the original probe spectrum which is monitored with the $165^{\circ}$ channel, (f), which detected the probe light scattered from the nozzle.

IAW has an angular spread similar to the Thomson satellites of the EPW. Such a large angular spread is indicative of the three-dimensional nature of the modulational instability, originating from the fact that the IAW is the decay product of the EPW, which itself has a small cross section (comparable to the plasma wavelength). Another important observation is that the position of the anti-Stokes peak varies with variation of angle.

Second, the temporal evolutions of the spectrum and the scattering efficiency were measured. If such scattering was indeed caused by the IAW, we can obtain the spatially averaged temporal evolution of the IAW amplitude by taking the square root of the Thomson satellite signal. Figure 13 shows the scattered spectrum around the probe wavelength for various probe delays at $25^{\circ}$-angle, $3 \mathrm{TW}$ laser power, and 3.7 $\times 10^{19} \mathrm{~cm}^{-3}$ plasma density. As can be seen, the temporal evolution of the Stokes and anti-Stokes peaks has features similar to that of the EPW, which is shown in Fig. 10. That is, the Stokes satellite is visible only when the probe delay is near the maximum of the anti-Stokes satellite, and in the case of the EPW, the opposite occurs. Figure 14 shows the scattering efficiency (the ratio between the intensities of the scattered light and the incident beam) of the probe pulse in the $25^{\circ}$ direction as a function of probe delay. The intensity of the Thomson satellite increases from almost zero (at zero delay) to the maximum (at about $T+20 \mathrm{ps}$ ) and then decreases gradually. Noteworthy is that in both cases the temporal evolution of the scattering efficiency is almost identical (reaching their peaks at the same time), even though their pump laser powers are significantly different.

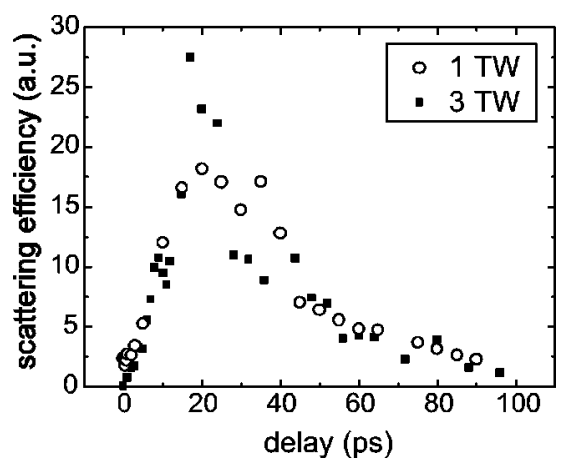

FIG. 14. Temporal evolution of the scattered intensity of the probe near the probe wavelength for various laser powers at $25^{\circ}$ and a plasma density of $3.7 \times 10^{19} \mathrm{~cm}^{-3}$.

Third, the spatio-temporal distribution of the probe scattering was measured. Figure 15 shows the side images of the scattered probe light near the probe wavelength, obtained using a narrow-bandpass filter that covers both the Stokes and anti-Stokes satellites, for various probe delays. The temporal evolution of the scattered intensity at any position along the channel was found to be roughly the same as that observed by the fiber array in the forward directions. This is similar to the behavior of the collective Thomson satellites from the EPW.

Fourth, although one may think that the spectra in Figs. 12 and 13 can be interpreted as the probe light scattered from the expanding plasma channel, two observations oppose such a possibility. First, the strongly enhanced scattering of the probe pulse at later delays (peaked at $T+20 \mathrm{ps)}$ was observed even when the laser power was very low (e.g., 0.3 TW) and no plasma-density depression was observed. Second, the expansion of the plasma channel has a higher velocity at a higher laser power, as observed in interferograms (see Ref. 25); however, we observed that the peak of the probe scattering occurs at about $T+20$ ps delay regardless of laser power (Fig. 14). Note that such invariance of the growth rate of the IAW with variation of laser power seems to be consistent with the invariance of the decay rate of the EPW.
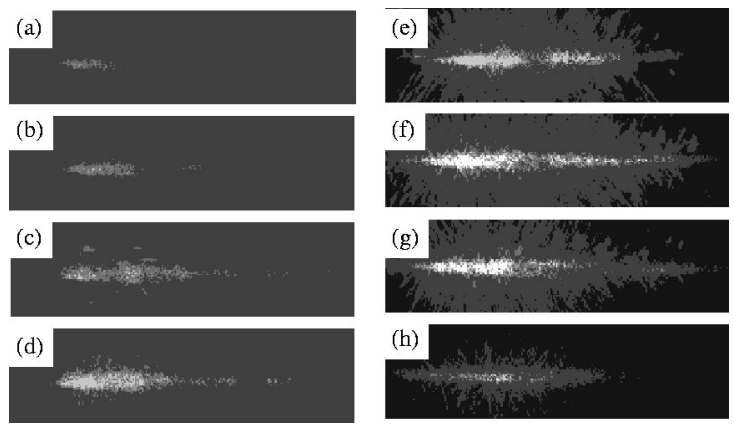

FIG. 15. Side imaging of the scattered intensity near the probe wavelength for various probe delays at $2.5 \mathrm{TW}$ laser power and $3.7 \times 10^{19} \mathrm{~cm}^{-3}$ plasma density: (a) $+0.3 \mathrm{ps}$, (b) $+0.9 \mathrm{ps}$, (c) $+1.9 \mathrm{ps}$, (d) $+3.4 \mathrm{ps,} \mathrm{(e)}+10 \mathrm{ps,} \mathrm{(f)}$ $+20 \mathrm{ps}$, (g) $+40 \mathrm{ps}$, (h) $+60 \mathrm{ps}$. The laser pulses propagate from left to right. 


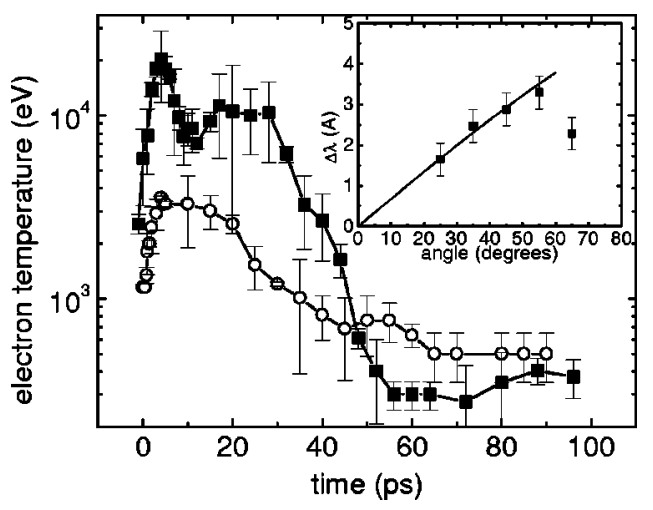

FIG. 16. Temporal evolution of the electron temperature for pump powers of $1 \mathrm{TW}$ (open circles) and $3 \mathrm{TW}$ (solid squares) at a plasma density of $3.7 \times 10^{19} \mathrm{~cm}^{-3}$. The inset shows the wavelength shift of the scattered probe light at various angles for the case of Fig. 12. The solid line satisfies the phase matching condition for the scattering of on-axis probe wave vector by ion acoustic waves propagating at a velocity consistent with an effective electron temperature of $3 \mathrm{keV}$.

In other words, all these observations for the scattering of the probe light to the spectral region near the probe wavelength are similar to those for the scattering of the probe pulse by the electron plasma wave, which are described in Secs. IV and V. Therefore, it is very likely that such scattering is indeed a result of collective Thomson scattering by ion acoustic waves. The fact that the growth of the IAW occurs after the pump-pulse passes the region of observation, indicates that the IAW observed seems to be a result of decay of the EPW through modulational instability. Although the EPW decays to almost zero within 3 ps after the passage of the pump pulse, the IAW continues growing until 20 ps later. This may be attributed to a cascading decay process. When the original EPW decays, its energy is converted to IAWs and mainly daughter EPWs. Because the modulational instability grows from ion-density fluctuation, which has a wide spread in phase space, and the EPW is three-dimensional in nature, the original EPW decays into a broad spectrum (and angular spread) of IAWs and EPWs. These daughter EPWs continue to cascade into IAWs and EPWs, and so on. This might explain why the IAW can continue to grow even though the original EPW is gone. Such a process can lead to strong Langmuir turbulence in the plasma. ${ }^{3}$ Eventually, all the energy of the original EPW is converted into that stored in the IAWs and the background plasma temperature (heated by warm wave breaking of the slow-phase-velocity daughter EPWs).

\section{Plasma heating}

The existence of an IAW can be used to diagnose the plasma temperature. The frequency shift of the Thomson scattering satellites of the IAW varies with angle because of the phase-matching conditions. The inset in Fig. 16 shows the wavelength shifts of the anti-Stokes satellites, with respect to the probe wavelength, scattered towards various directions for the case of Fig. 12. The fitting line is from the phase-matching condition for the scattering of the on-axis probe wave vector by the IAWs, $\mathbf{k}_{s}-\mathbf{k}_{i}=\mathbf{k}_{\mathrm{IAW}}$, where $\mathbf{k}_{s}$, $\mathbf{k}_{i}$, and $\mathbf{k}_{\mathrm{IAW}}$ are the wave vectors of the anti-Stokes satellite, the probe and the IAW, respectively. Note that $\mathbf{k}_{s}$ is determined by the direction (angle) of observation and $\mathbf{k}_{\mathrm{IAW}}$ is determined by the IAW frequency $\left(\omega_{\mathrm{IAW}}\right)$ and the sound speed $\left(c_{s}\right)$, i.e., $\omega_{\mathrm{IAW}}=k_{\mathrm{IAW}} \cdot c_{s}$. Therefore, by measuring the anti-Stokes frequency shift (equal to the IAW frequency) at a specific angle, the sound speed can be obtained and the plasma electron temperature can be determined. A very good match is made when the effective electron temperature is set to be $3 \mathrm{keV}$. (Here we use $c_{s}=\sqrt{T_{e} / m_{i} /\left(1+k_{\mathrm{IAW}}^{2} \lambda_{e}^{2}\right)}$, where $m_{i}$ is the ion mass and $\lambda_{e}\left(T_{e}\right)$ is the electron Debye length, to define the effective electron temperature $T_{e}$ because, e.g., the electron energy distribution may not be Maxwellian.) The satisfaction of this phase-matching condition is consistent with collective Thomson scattering by IAWs. The deviation of the Thomson satellite wavelength from the fitting line at large angles is probably due to the fact that the scattered signals in these directions might be dominated by the scattering of the off-axis probe wave vectors. By finding the right temperature to fit the anti-Stokes frequency shift at small angles, we can obtain the effective electron temperature at various probe delays. ${ }^{46}$ Figure 16 shows the temporal evolution of the effective electron temperature. The effective electron temperature increases to $20 \mathrm{keV}(3 \mathrm{keV})$ at $3 \mathrm{ps}$ after the passage of the pump-pulse for a pump power of 3 TW (1 TW), and then drops to around $400 \mathrm{eV}$ after $50 \mathrm{ps.}$

Note that the effective electron temperature increases while the original EPW damps and that the position of the maximum electron temperature coincides with the complete extinction of the original EPW (at $T+3 \mathrm{ps)}$ ). Furthermore, a $\Delta n / n_{0}=0.3(0.1) \mathrm{EPW}$ with a phase velocity close to the speed of light has an average electron energy $\left(\frac{1}{2} m_{e} u_{e}^{2}\right.$, where $u_{e}$ is the peak fluid velocity of electrons in the EPW) of 23 $\mathrm{keV}(2.5 \mathrm{keV})$. This is roughly equal to the effective electron temperature measured right after the decay of the EPW. These observations seem to indicate that the energy stored in the original EPW is transferred to IAWs and, mainly, the effective electron temperature, upon its decay. The model of modulational instability decay shows that, when the original EPW damps, the energy from the original EPW must be converted into mainly the energy stored in the daughter EPWs. Therefore, the effective electron temperature we measured is probably the average electron energy stored in both the turbulent motion of electrons (composed of the daughter EPWs generated during the three-dimensional cascading process) and the random thermal motion of electrons. At early delays (right after the decay of the original EPW), the turbulent motion of electrons dominates the effective electron temperature. Gradually, more and more energy is converted to the random thermal motion of electrons by way of warm wave breaking of daughter EPWs. At $T+20 \mathrm{ps}$, the daughter EPWs are exhausted so that the IAWs cease to grow and the energy is mainly stored in the random thermal motion of electrons. The cooling of the effective electron temperature may result from thermal conduction and hydrodynamic expansion. 


\section{SUMMARY}

In this experiment, the spatially, temporally, and angularly resolved Raman and collective Thomson scattering spectra were used to study the growth and decay of a selfmodulated laser wakefield. As the laser pulse propagated in the plasma, an electron plasma wave was excited through Raman forward scattering and self-modulation instabilities, as is evident from the appearance of spectral cascading of Raman satellite. The angular spread of the forward Raman satellites was observed to be larger than the transmitted laser beam, indicating that this Raman forward scattering process is three-dimensional in nature. The spatially averaged temporal evolution of the electron plasma wave excited was measured using collective Thomson scattering of a probe pulse, showing an exponential growth and decay. Side imaging of the collective Thomson scattering shows that such spatially averaged result can describe the temporal evolutions of the electron plasma waves at different positions along the plasma-wave channel.

The change of the decay rate (and the duration) of the electron plasma wave when the laser power was varied was observed to be insignificant, even when the number of electrons trapped and accelerated by the electron plasma wave varied from zero to $10^{10}$. This indicates that there are other damping mechanisms that are important in our experiment in addition to electron beam loading. Our observation of the spatially, temporally, and angularly resolved collective Thomson scattering spectra, in the vicinity of the probe wavelength, seems to support the modulational instability decay of the electron-plasma wave as the main damping mechanism. Under such assumption, the collective Thomson scattering satellites scattered from ion acoustic waves can be used to diagnose the temporal evolution of the plasma temperature. The heating of the plasma measured using this method seems to be consistent with the decay of the electron plasma wave as a result of modulational instability.

Production of cold plasmas, through short-pulse tunneling ionization, is considered crucial for the success of the proposed recombinational $\mathrm{x}$-ray lasers in the transient regime. ${ }^{47}$ The electron temperature of plasmas produced by short intense laser pulses was previously measured by Glover et al. ${ }^{48}$ and found to be consistent with the prediction of the tunneling ionization model. ${ }^{49}$ Inverse bremsstrahlung heating is small in this case due to short pulse duration and large electron quiver velocity. However, in circumstances where EPWs are excited (due to resonant or self-modulated laser wakefields), it was predicted that the damping of EPWs can lead to additional rapid heating of the plasma, causing breakdown of the lasing conditions. ${ }^{47}$ Therefore, the process of plasma heating via excitation and damping of EPWs must be understood in order to control this problem. On the positive side, this plasma heating process might provide a way to heat high-density plasmas for applications such as shortwavelength collisionally pumped $\mathrm{x}$-ray lasers ${ }^{50}$ and nuclear fusion. ${ }^{1}$

\section{ACKNOWLEDGMENTS}

This work was supported by the Division of High Energy Physics, Office of Energy Research, U.S. Department of
Energy, and the National Science Foundation. We would also like to thank E. Dodd, M. C. Downer, P. Drake, J.-K. Kim, S. P. Le Blanc, G. Mourou, and R. Wagner for their useful discussions.

${ }^{1}$ M. Tabak, J. Hammer, M. E. Glinsky, W. L. Kruer, S. C. Wilks, and R. J. Mason, Phys. Plasmas 1, 1626 (1994).

${ }^{2}$ E. Esarey, P. Sprangle, J. Krall, and A. Ting, IEEE Trans. Plasma Sci. PS-24, 252 (1996).

${ }^{3}$ V. E. Zakharov, Sov. Phys. JETP 35, 908 (1972).

${ }^{4}$ D. Pesme, S. J. Karttunen, R. R. E. Salomaa, G. Laval, and N. Silvestre, Laser Part. Beams 6, 199 (1988).

${ }^{5}$ P. Mora, D. Pesme, A. Héron, G. Laval, and N. Silvestre, Phys. Rev. Lett. 61, 1611 (1988).

${ }^{6}$ R. P. Drake and S. H. Batha, Phys. Fluids B 3, 2936 (1991).

${ }^{7}$ D. M. Villeneuve, K. L. Baker, R. P. Drake, B. Sleaford, B. La Fontaine, K. Estabrook, and M. K. Prasad, Phys. Rev. Lett. 71, 368 (1993).

${ }^{8}$ R. P. Drake, R. E. Turner, B. F. Lasinski, K. G. Estabrook, E. M. Campbell, C. L. Wang, D. W. Phillion, E. A. Williams, and W. L. Kruer, Phys. Rev. Lett. 53, 1739 (1984).

${ }^{9}$ C. Darrow, D. Umstadter, T. Katsouleas, W. B. Mori, C. E. Clayton, and C. Joshi, Phys. Rev. Lett. 56, 2629 (1986).

${ }^{10}$ F. Amiranoff, M. Laberge, J. R. Marquès, F. Moulin, E. Fabre, B. Cros, G. Matthieussent, P. Benkheiri, F. Jacquet, J. Meyer, P. Miné, C. Stenz, and P. Mora, Phys. Rev. Lett. 68, 3710 (1992).

${ }^{11}$ K.-C. Tzeng, W. B. Mori, and T. Katsouleas, Phys. Rev. Lett. 79, 5258 (1997).

${ }^{12}$ D. Umstadter, S.-Y. Chen, A. Maksimchuk, G. Mourou, and R. Wagner, Science 273, 472 (1996).

${ }^{13}$ C. D. Decker, W. B. Mori, and T. Katsouleas, Phys. Rev. E 50, R3338 (1994).

${ }^{14}$ P. Bertrand, A. Ghizzo, S. J. Karttunen, T. J. H. Pattikangas, R. R. E. Salomaa, and M. Shoucri, Phys. Rev. E 49, 5656 (1994).

${ }^{15}$ E. Esarey, B Hafizi, R. Hubbard, and A. Ting, Phys. Rev. Lett. 80, 5552 (1998).

${ }^{16}$ S. V. Bulanov, F. Pegoraro, A. M. Pukhov, and A. S. Sakharov, Phys. Rev. Lett. 78, 4205 (1997).

${ }^{17}$ S. P. Le Blanc, M. C. Downer, R. Wagner, S.-Y. Chen, A. Maksimchuk, G. Mourou, and D. Umstadter, Phys. Rev. Lett. 77, 5381 (1996).

${ }^{18}$ A. Ting, K. Krushelnick, C. I. Moore, H. R. Burris, E. Esarey, J. Krall, and P. Sprangle, Phys. Rev. Lett. 77, 5377 (1996).

${ }^{19}$ N. E. Andreev, L M. Gorbunov, V. I. Kirsanov, A. A. Pogosova, and R. R. Ramazashvili, Phys. Scr. 49, 101 (1994).

${ }^{20}$ N. E. Andreev, L. M. Gorbunov, and R. R. Ramazashvili, Plasma Phys. Rep. 23, 277 (1997).

${ }^{21}$ E. Esarey, J. Krall, and P. Sprangle, Phys. Rev. Lett. 72, 2887 (1994).

${ }^{22}$ W. B. Mori, C. D. Decker, D. E. Hinkel, and T. Katsouleas, Phys. Rev. Lett. 72, 1482 (1994).

${ }^{23}$ C. D. Decker, W. B. Mori, T. Katsouleas, and D. E. Hinkel, Phys. Plasmas 3, 1360 (1996).

${ }^{24}$ R. Wagner, S.-Y. Chen, A. Maksimchuk, and D. Umstadter, Phys. Rev. Lett. 78, 3125 (1997).

${ }^{25}$ S.-Y. Chen, G. S. Sarkisov, A. Maksimchuk, R. Wagner, and D. Umstadter, Phys. Rev. Lett. 80, 2610 (1998).

${ }^{26}$ K. Krushelnick, A. Ting, C. I. Moore, H. R. Burris, E. Esarey, P. Sprangle, and M. Baine, Phys. Rev. Lett. 78, 4047 (1997).

${ }^{27}$ A. Modena, Z. Najmudin, A. E. Dangor, C. E. Clayton, K. A. Marsh, C. Joshi, V. Malka, C. B. Darrow, C. Danson, D. Neely, and F. N. Walsh, Nature (London) 377, 606 (1995).

${ }^{28}$ M. J. Everett, A. Lai, D. Gordan, K. Wharton, C. E. Clayton, W. B. Mori, and C. Joshi, Phys. Rev. Lett. 74, 1355 (1995).

${ }^{29}$ J. F. Drake, P. K. Kaw, Y. C. Lee, G. Schmidt, C. S. Liu, and M. Rosenbluth, Phys. Fluids 17, 778 (1974).

${ }^{30}$ W. P. Leemans, C. E. Clayton, K. A. Marsh, and C. Joshi, Phys. Rev. Lett. 91, 1434 (1991).

${ }^{31}$ C. B. Darrow, C. Coverdale, M. D. Perry, W. B. Mori, C. Clayton, K. Marsh, and C. Joshi, Phys. Rev. Lett. 69, 442 (1992).

${ }^{32}$ R. E. Slusher and C. M. Surko, Phys. Fluids 23, 472 (1980).

${ }^{33}$ D. Umstadter, R. Williams, C. Clayton, and C. Joshi, Phys. Rev. Lett. 59, 292 (1987).

${ }^{34}$ E. A. Jackson, Phys. Fluids 3, 831 (1960).

${ }^{35}$ P. Koch and J. Albritton, Phys. Rev. Lett. 34, 1616 (1975). 
${ }^{36}$ L. S. Kuzmenkov, A. A. Sokolov, and O. O. Trubachev, Sov. Phys. J. 26, 1076 (1983).

${ }^{37}$ J. M. Dawson, Phys. Rev. 113, 383 (1959).

${ }^{38}$ A. I. Akheizer and R. V. Polovin, Sov. Phys. JETP 3, 696 (1956).

${ }^{39}$ T. C. Chiou, T. Katsouleas, and W. B. Mori, Phys. Plasmas 3, 1700 (1996).

${ }^{40}$ C. S. Liu and V. K. Tripathi, Phys. Plasmas 3, 3410 (1996).

${ }^{41}$ A. McPherson, A. B. Borisov, K. Boyer, and C. K. Rhodes, J. Phys. B 29, L291 (1996).

${ }^{42}$ W. B. Mori and T. C. Katsouleas, Phys. Rev. Lett. 69, 3495 (1992).

${ }^{43}$ P. Sprangle, E. Esarey, and A. Ting, Phys. Rev. B 41, 4463 (1990).
${ }^{44}$ P. Sprangle, E. Esarey, and A. Ting, Phys. Rev. Lett. 64, 2011 (1990).

${ }^{45}$ A. Pukhov and J. Meyer-ter-Vehn, Phys. Rev. Lett. 76, 3975 (1996).

${ }^{46}$ C. Laubaune, H. A. Baldis, N. Renard, E. Schifano, S. D. Baton, A. Michard, W. Seka, R. E. Bahr, B. S. Bauer, K. Baker, and K. Estabrook, Phys. Rev. Lett. 75, 248 (1995).

${ }^{47}$ P. Amendt, D. C. Eder, and S. C. Wilks, Phys. Rev. Lett. 66, 2589 (1991).

${ }^{48}$ P. E. Glover, T. D. Donnelly, E. A. Lipman, A. Sullivan, and R. W. Falcone, Phys. Rev. Lett. 73, 78 (1994).

${ }^{49}$ P. B. Corkum, N. H. Burnett, and F. Brunel, Phys. Rev. Lett. 62, 1259 (1989).

${ }^{50}$ R. C. Elton, X-ray lasers (Academic, San Diego, CA, 1990). 